\title{
August Forel und Dumeng Bezzola - ein Briefwechsel
}

\author{
herausgegeben und kommentiert von Christian Müller
}

\section{Zusammenfassung}

Der vorliegende Beitrag stellt einen kleinen Ausschnitt aus der reichen epistolarischen Tätigkeit August Forels (1848-1931) dar. Es handelt sich um den Briefwechsel mit dem seither etwas in Vergessenheit geratenen Psychiater Dumeng Bezzola (1868-1936). In Forels Briefen (aus den Jahren 1901-1916) kommt seine ambivalente Einstellung gegenüber Freud zum Ausdruck. Sie überträgt sich auch auf seinen eigenen Nachfolger am Zürcher Burghölzli, Eugen Bleuler, und dessen Mitarbeiter Carl Gustav Jung, die sich beide darum bemühten, die Psychoanalyse für die Klinik der Gemüts- und Geisteskrankheiten fruchtbar zu machen.

Es kann hier nicht darum gehen, auf die Person und das Wirken August Forels (1848-1931) einzugehen. Ich setze voraus, daß der Leser in großen Zügen beides kennt. Nützlich war mir für die Bearbeitung dieses Briefwechsels die großartige Briefausgabe von H. Walser ${ }^{8}$, Forels Selbstbiographie ${ }^{6}$, das Buch von A. Wettley ${ }^{9}$ sowie Forels Buch über Hypnotismus ${ }^{5}$.

Dagegen soll der Briefpartner Dumeng Bezzola etwas ausführlicher ins Licht gerückt werden. Ich stütze mich dabei vor allem auf den Nekrolog von F. Braun ${ }^{4}$ sowie auf einen umfangreichen Nachlaß mit zahlreichen persönlichen Dokumenten Bezzolas, der gegenwärtig im Berner Medizinhistorischen Institut der Bearbeitung harrt. (Herrn Dr. Rudolf Campell in Pontresina sei an dieser Stelle herzlich dafür gedankt, daß er diesen Nachlaß der Wissenschaft zur Verfügung gestellt hat.)

Dumeng Bezzola wurde 1868 in Zernez geboren. Das Gymnasium besuchte er in Winterthur. Früh schon interessierte er sich für Botanik und legte ein auch später beachtetes Herbarium der Pflanzen des Nationalparkes an. Er studierte Medizin in Zürich, Genf und Heidelberg, wurde Assistent bei Heinrich Bircher in Aarau und wandte sich dann der Psychiatrie zu. Seit 1896 wirkte er als Sekundärarzt in der kantonalen Heilanstalt Waldhaus bei Chur. Später übernahm er unter der Direktion von Ludwig Frank (1863-1935) die ärztliche Leitung eines privaten Sanatoriums Schloß Hard im Thurgau. Dort blieb er bis 1909 und setzte sich aktiv für die Behandlung 
und Bekämpfung des Alkoholismus ein. Zugleich begann er sich für die Gedanken des Kreises um Freud und Breuer in Wien zu interessieren. Mit Bleuler, Jung, Bertschinger, Frank kann er zu den ersten Schweizer Ärzten gezählt werden, die aufmerksam die neue Bewegung verfolgten und auch die therapeutische Methodik anzuwenden suchten. Später lehnte er allerdings mehr und mehr die Theorien Freuds ab und versuchte einen eigenen Weg zu gehen, indem er weiterhin - wie Breuer es getan hatte - die Hypnose als Ausgangspunkt seines therapeutischen Handelns nahm. Auch E. Bleuler und C.G.Jung stand er kritisch gegenüber. Nach seinem Weggang von Schloß Hard, wo seine Tätigkeit ihn in Konflikte mit L. Frank gebracht hatte, was viel Bitterkeit in ihm auslöste, zog er ins Engadin zurück und wirkte dort bis zu seinem Tode (1936) als Nervenarzt. Aus seinem Nachlaß geht hervor, daß er eine rege Tätigkeit entfaltete und eine internationale Klientel behandelte. Bekannte Nervenärzte aus Deutschland und Frankreich wiesen im Patienten zu. Er war offenbar aber auch ein beliebter Praktiker, der sich um die Gesundheitspolitik seiner Region kümmerte. In unermüdlichem Eifer notierte er täglich alle seine Beobachtungen und führte genauestens Tagebuch.

Wie kam es zum Briefwechsel mit Forel? Dieser hatte 1898 seine Professur in Zürich aufgegeben und sich nach Chigny bei Morges zurückgezogen. Als unermüdlicher Kämpfer gegen den Alkoholismus mußte Forel Gefallen finden an der eindeutigen, kompromißlosen Einstellung Bezzolas dem Alkoholproblem gegenüber. Ein weiteres verbindendes Element zwischen den beiden Männern war wohl ihr Bestreben, psychotherapeutisch neue Wege zu gehen, wobei sie sich aber einig fanden in der Ablehnung der Jungschen und Freudschen Auffassungen. A. Forel hat D. Bezzola immer wieder Alkoholkranke zur Behandlung überwiesen und ein guter Teil der vorliegenden Briefe bezieht sich auf sie. Bezzola wird übrigens in verschiedenen Briefen Forels, die sich in Walsers Briefausgabe befinden, erwähnt. Ebenfalls finden wir seinen Namen mehrmals im Briefwechsel JungFreud $^{7}$, allerdings erscheint er dort in einem wenig vorteilhaften Licht.

In der Autobiographie Forels ${ }^{6}$ und im Buch von A. Wettley ${ }^{9}$ wird Bezzola nicht erwähnt.

Es liegen im Nachlaß Bezzolas insgesamt 21 Briefe und 16 Postkarten August Forels vor. Sie sollen hier in chronologischer Reihenfolge wiedergegeben werden. Die am wenigsten interessanten Passagen, die z. B. unbekannte Patienten betreffen, sollen dabei ausgelassen werden. Ebenso habe ich darauf verzichtet, alle erhaltenen Kopien von Antworten Bezzolas hier 
zu publizieren. Der wohl wichtigste Brief Bezzolas an Forel von 1911 ist in der Walserschen Briefsammlung (S.411) bereits veröffentlicht. Abgesehen von den hier publizierten Gegenbriefen Bezzolas (Nr. 23, 24, 34, 37, 39) liegen im Medizinhistorischen Institut Zürich noch drei Gegenbriefe Bezzolas unbedeutenden Inhalts.

\section{Handschriftlicher Brief}

Sehr geehrter Herr College,

Chigny, 8.03.1901

Beiliegend Ihre Formulare - Ihr «Übersetzer» hatte ein mustergültiges «français fédéral» zusammengestoppelt. Es wird nötig sein, daß mein corrigiertes Manuskript, so unsauber es auch ist, direct der Druckerei übergeben wird, da sonst der Abschreibende Fehler hinein corrigieren wird (alte Erfahrung). Wenn Sie die Sache der Univ.-Buchdruckerei Fritz Reinhard, 15 St-Albanstraße, Basel, schicken, wird es ganz gut gehen, da meine Schrift dort gut bekannt ist.

Es wird dennoch absolut nötig sein, daß mir eine Correctur mit Manuscript geschickt wird.

Ich habe Ihre Ansicht, die ich durchaus teile, daß die alkoholfreie Behandlung Nervenkranker zu betonen sei mehr als die Nervenbehandlung der Alkoholiker, dabei vor Augen gehabt und die Ellikoner Rubriken darnach etwas modifiziert. Vielleicht wünschen Sie es noch mehr, dann sagen Sie es mir. Doch würde dies wohl weniger im ärztlichen Formular als in einem besonderen Prospekt zu betonen sein.

Herzlichen Gruß und hoffentlich auf Wiedersehen in Genf am Pfingstmontag.

Ihr Forel

Dieser erste Brief Forels läßt vermuten, daß Bezzola ihm seine «übersetzte» Arbeit, die er 1901 in Wien vortrug, zur Überprüfung vorlegte. Zugleich hat wohl Bezzola um statistische Unterlagen aus Ellikon (von Forel gegründete Zürcher Heilstätte für Alkoholkranke) gebeten. 


\section{Postkarte}

Lausanne, 21.11.1901

Forel bittet Bezzola, einen Prospekt des Sanatoriums Hard an einen Kollegen in Lausanne zu schicken, der einen Patienten durch B. behandeln lassen möchte.

\section{Postkarte}

Hochgeehrter Herr College,

Chigny, 27.11.1901

Da Sie nicht antworteten, mußte ich in dem bereits eingesandten Manuskript über Schloß Hard f. d.Schw. Lexikon mit den Angaben von Marthalers Tableau Vorlieb nehmen.

Forboro ist nicht ein Mann sondern eine Anstalt (für Trinker), das Beste in den U.S. Dort werden Turnübungen sowie Wägungen und Lungencapacitätsmessungen beim Eintritt und Austritt der Patienten vorgenommen, welche ungeheuer günstig wirken auf Patienten und Angehörige. Der Turnlehrer meint, es käme vom Turnen allein, ich aber sage zum großen Teil auf Abstinenz. Doch ist das Turnen etc. ausgezeichnet. Ich gab die genaue Adresse schon vor 1 1 $1 / 2$ Jahren an Frank und Liebekranz [? schwer leserlich] und wundere mich, daß man auf Schloß Hard weder Notiz davon nahm, noch dahin schrieb. Schreiben Sie an: Mrs Anna Phillips Williams, 505 Beaconstreet, Boston, Mass., USA und bitten sie um die Jahresberichte und System vom Turnen, Arbeiten, etc. in Forboro (Wenn sie nicht antworten an Prof. Koren, 784 Bacon Street, Boston, Mass) und empfehlen Sie sich von mir. - Ihre wissenschaftlichen Arbeiten sind sehr interessant, besonders Ihre teilweise Bestätigung Smiths. Bezüglich Periodicität kann ich ja die Sache nicht beurteilen. - Etwas zu metaphysisch scheint mir Ihre [unleserlich] Speculation. Aber die Tatsachen sind sehr interessant. Sie können allein beurteilen wieweit dieselben konstant sind. Jetzt jedoch tut es Not Schloß Hard mehr in Credit zu bringen, daß es besser besucht wird. Und da scheinen mir gute Jahresberichte mit Ergebnissen à la Forboro und Ellikon, wie ich es dort und auch im «Burghölzli» grosso modo einführte, sehr nötig. Denn es liegt rein nichts Schriftliches vor und es hüllte sich Schloß Hard in Dunkel. Was Sie zuletzt schreiben bezüglich suggestiven Einfluß auf die Alkoholiker unterschreibe ich mit beiden Händen.

Herzlichen Gruß Ihr Forel 


\section{Postkarte}

St. Gallen, 4.04. 1902

Forel kündigt seinen Besuch in Schlo $\beta$ Hard an; erwähnt, daß er am Abend einen Vortrag in Konstanz halten werde.

\section{Handschriftlicher Brief}

Chigny, 22.4. 1902

Lieber Herr College,

Herr D. kam gestern total besoffen zu mir. Er war von seinem Vater und Dr. B. in Rom mir empfohlen. Zu seiner Behandlung war mir Vollmacht gegeben. Der Mann ist bereits leber- und herzkrank, nicht weit vom Delirium, sauft Cognac und verbringt die Nächte in Genf in Bordellen.

Ich habe ihn gezwungen eine Nacht hier zu bleiben um seinen Rausch auszuschlafen. Heute früh habe ich ihn untersucht. Er war weich und versprach mir in die Hand freiwillig zu Ihnen nach Schloß Hard zu gehen und dort 6-8 Monate zu bleiben. Er kann englisch und einige Worte französisch.

Leider mußte ich ihn heute nach Genf zurückgehen lassen, wo er alle seine Sachen hat. Natürlich wird er dort wieder saufen. Ich ersuche Sie nun auf Grund dieser Zeilen ihn aufzunehmen. Er hat 10000 Dollars (50000 Fr.) Einkommen jährlich, kann also 15 Fr. oder 20 Fr. und mehr täglich bezahlen. Nüchtern ist er wohl gebildet und recht intelligent. Er hat selber durch Arbeit sein Vermögen erworben, sich aber dann vor 6 Jahren zur Ruhe gesetzt und dann sich mit Bacchus und Venus ruiniert. Der Vater ist unglücklich und möchte ihn retten. Ich habe Schloß Hard als unbedingte und einzige conditio sine qua non der Heilung hingestellt. Vielleicht hält er sein Versprechen und geht freiwillig hin, sonst wird wohl die Familie dazu helfen.

Diagnose: Schwerer Fall von chronischem Alkoholismus. Behandlung von 6-12 Monaten in einer Trinkerheilanstalt absolut erforderlich.

Mit collegialem Gruße Prof. Dr A. Forel 


\section{Postkarte}

Chigny, 10.05.1902

Forel meldet, da $\beta$ der obenerwähnte Patient durchgebrannt und übrigens ein hoffnungsloser Fall sei. Erwähnt eine Frage Bezzolas betreffend Selbstkritik und verspricht ihm seinen Rat. Lobt eine norwegische Arbeit (über Alkoholismus), stellt fest, da $\beta$ in Norwegen die Abstinenten "fast in Mehrheit sind». Fragt sich, wie es mit dem Geisteszustand abstinenter Hundertjähriger stehe.

\section{Eigenhändiger Brief}

Lieber Herr College,

Chigny, 16.06.1904

darf ich Sie bitten, an Herrn Prof. Hercod in Lausanne (secrétariat antialcoolique Lausanne) die letzten Jahresberichte u. Bilanzen von Schloß Hard zuzusenden. Es soll im Ct Waadt auf ähnlicher Basis etwas anderes, nämlich ein Erholungssanatorium (nicht für Trinker) auf abstinenter Basis gegründet werden und Dr Loy - Hr Hercod möchten sehen, wie man es in Schloß Hard macht.

Herzlichen Gruß von Haus zu Haus und Dank zum Voraus

Hercod und Loy sind bei Walser nicht erwähnt. Robert Hercod, geb. 1876, war Generalsekretär des antialkoholischen Vereins.

\section{Postkarte}

Chigny, 21.12.1904

Forel hat einem Patienten Schloß Hard empfohlen. «Es ist ein herzzerreißender Fall».

\section{Postkarte}

Chigny, 18.031905

Forel meldet, daß sein Haus «voll ist wie ein Ei ohne Hohlraum» und daß er niemanden mehr aufnehmen kann. Empfiehlt für eine Patientin von Bezzola Dr. Vittoz in Lausanne, der sich eben etabliert habe und der «für den Hypnotismus sich spezialisiert hat und verschiedene Kranke von mir vortrefflich behandelt hat». F. werde abwesend sein, um Vorträge in Langnau, Langenthal etc. zu halten. 


\section{Postkarte}

Chigny, 19.03.1905

F. meldet Änderung der Pläne, will die Patientin in Chigny empfangen.

\section{Postkarte}

Chigny, 26.09.1905

Lieber Herr College,

in Chigny zurückgekehrt finde ich daß meine liebe Frau in der That ein Ausspannen sehr nötig hat, wobei sie volle Ruhe und Allein sein in der Natur nötig hat. Ich hoffe, sie zu bestimmen, bald nach Schloß Hard zu gehen. Paßt es Ihnen noch, so bitte ich um eine Postkarte. Sie wird zugleich mich vorteilhaft als Experte stellvertreten.

\section{Mit freundlichem Gruß an Sie Ihr Forel}

\section{Eigenhändiger Brief}

Chigny, 8.10.1905

F. empfiehlt die Aufnahme eines russischen Fürsten, Alkoholiker, auf Schloß Hard. Beschreibt seinen Fall und fügt schließlich bei, der Patient sollte Guttempler werden, sollte die Alkoholfrage studieren, Ellikon besuchen und in seiner Heimat zum Besten des Vaterlandes öffentlich wirken.

\section{Postkarte}

30.11 .1905

F. dankt für eine Belehrung und meldet, daß er an zuständigem Ort interveniert habe.

\section{Eigenhändiger Brief}

Chigny, 15.01.1906

F. meldet B. einen Patienten aus Yverdon an. «Er sollte der neuen Loge beitreten, die wir in Yverdon-Grandson gründen wollen». 


\section{Eigenhändiger Brief}

Forel empfiehlt einen Patienten zur Aufnahme ins Schlo $\beta$ Hard. «Ich habe bei mir keinen Platz und bin zu Tode gehetzt».

Forel nahm in Chigny auch einzelne Patienten bei sich zur Behandlung auf.

\section{Eigenhändiger Brief}

Chigny, 30.06.1906

Forel empfiehlt wieder einen Patienten, der außer Alkoholismus Zeichen einer progressiven Paralyse zeige. Beschreibt die neurologischen Zeichen. «Ich möchte Ihnen dringend empfehlen behufs Behandlung solcher Fälle (Metasyphilis incip. - Tabes-Paralyse) sich mit Dr Carl (nicht Lucius) Spengler in Davos betreffend Jothion Behandlung in Verbindung zu setzen. Ich weiß genau, daß Turban und seine Klicke alles aufbieten um Carl Spenglers Erfolge und bakteriologischen Arbeiten schlecht zu machen. Was ich aber weiß und gesehen habe macht, daß ich mich nicht beirren lasse. Unsere Freundin, Frau Prof. Fuchs von Wolfring aus Wien, eine der tüchtigsten Ärztinnen die ich kenne, besonders im Mikroskopieren und Histologie und überhaupt - arbeitet jetzt bei Carl Spengler.

Der Zusammenhang der Metasyphilis mit der Tuberkulose beruht auf dem Residualvorgang der Bakterien. [...]»

Der Name von Frau Prof. Fuchs findet sich bei Walser nicht.

\section{Postkarte}

Chigny, 9. XI. 1906

Lieber Herr College,

Besten Dank. Hetze unglaublich. Ich glaube kaum, daß ich nach Schloß Hard komme. Ihre Schlußsätze verstehe ich so in Globo ungefähr; doch gestehe ich, daß ich den Proceß der Reconstruction nie ganz klar habe verstehen und noch weniger ausführen können, trotz div. Versuche und trotz Frank's und Ihren Erklärungen. Ich mag die Leute auch noch so sehr im Wachen oder in der Hypnose zur Erinnerung an die alten Ereignisse mahnen, es will nichts dabei herauskommen (bei mir wenigstens)

Mit herzlichem Gruß Ihr Forel 
Bezzola betonte häufig, auch in seinen Publikationen, daß der Psychoanalyse die Psychosynthese folgen müsse, d.h. die Rekonstruktion.

\section{Postkarte}

Yvorne, d. 15.XI.07

Mein lieber Kollege,

Herzlichsten Dank für Ihren Brief. Sie werden uns stets herzlichst willkommen sein, aber nicht als Patient sondern als Freund!

Es tut mir außerordentlich leid, daß Sie von Schloß Hard fort wollen. Wie wird es dort gehen? Ich schreibe an Frank in dem von Ihnen gewünschten Sinn. Schicken Sie mir aber keine Protokolle denn ich bin - so sehr ich mich dafür interessieren würde - derart überarbeitet, daß ich verzweifle und nur zum dringendsten Zeit finde. Die erzählten Fälle interessieren mich aber sehr. Ich werde Ihnen also bald den Hervé schicken. Also wegen de Montet schreibe ich an Frank.

Mit herzlichstem Gruß von Haus zu Haus, Ihr Forel

19. Kopie eines Briefes an Ludwig Frank

Yvorne, d. 15.XI.07

Lieber Freund,

Herzlichsten Dank für Ihren lieben Empfang in Zürich. Meine Frau hat Ihrer Frau Gemahlin wegen den Quitten geschrieben.

Ich erfahre 2 Dinge über die ich mit Ihnen sprechen muß und von welchem einem Sie mir nichts sagten: Bezzola will ja von Schloß Hard fort! Das wäre ein Jammer. Zugleich ist der junge de Montet der sich in Genf bei Weber gemeldet hatte, durch den Genfer Protektionismus abgewiesen worden und man sagt sogar, daß Weber deswegen an Rücktritt denkt. (Sagten Sie mir Letzteres oder war es jemand anderes?) De Montet soll dadurch sehr entmutigt sein. Nun hat mir Bezzola sehr viel gutes von de Montet gesagt. Ich glaube, er würde ihn sehr gerne als Nachfolger haben. Es soll ein sehr tüchtiger junger Mann sein, der sich für die Psychanalyse sehr interessiert. Aber es scheint, daß er nicht mehr den Mut hat selbst zu 
candidieren, wegen der Abweisung in Genf. Es müßte der Verwaltungsrat von Schloß Hard ihn direkt anfragen, also den ersten Schritt tun. Ich bitte Sie dringend mit Bezzola darüber zu korrespondieren. Ich muß ganz offen sagen, daß es mir sehr unangenehm wäre, wenn jemand von der Jung-FreudKlicke von Burghölzli dahin käme, denn dann wäre es aus mit meiner Verbindung mit Schloß Hard, das sage ich ganz offen und gerade aus. Mich ekelt dieser Freudkultus an, genau so sehr wie Bezzola.

Ich will es dahin gestellt sein lassen, ob die berühmte Entdeckung Freud's wirklich ihm gehört und nicht viel mehr Breuer, aber sicher ist es, daß Freud sich in Wien, wo man doch sicher nicht zimperlich ist, einen recht schlechten Ruf gemacht hat und zwar durchaus nicht ohne Grund. Mit dieser Kombination von Größenwahn, Eigendünkel und Schweinerei genannt Freud, Jung und Cie, will ich nichts zu tun haben, das erkläre ich ganz kategorisch, um jeder Unklarheit für die Zukunft vorzubeugen.

Es kommt mir vor als ob Bleuler gar nicht mehr Direktor von Burghölzli wäre, sondern Jung, und es tut mir leid. Ich stehe da ganz auf der Seite von Bezzola.

Mit herzlichstem Gruß von Haus zu Haus Ihr Forel.

Der von Forel und Bezzola angesprochene Konflikt mit Frank wird aus der Korrespondenz Forel-Bezzola nicht deutlich. Im Archiv Bezzola im Medizinhistorischen Institut Bern liegt ein umfangreicher Briefwechsel FrankBezzola, der möglicherweise Aufschluß erteilen wird. Der erwähnte de Montet ist Charles de Montet, geb. 1880, von Vevey, Psychiater, später PD an der Universität Lausanne.

\section{Handschriftlicher Brief}

Yvorne, 18.11.1907

Lieber Herr College,

Frank klagt nun seinerseits, daß Sie sich ganz von ihm abwenden und nie mit ihm sprechen. Das ist ein Fehler, lieber Freund, Sie haben gegen Frank eine ganz ungerechtfertigte Pike, die ich längst kenne. Im Interesse der Sache wie in Ihrem eigenen bitte ich Sie dringend, sich einmal gründlich und freundlich mit ihm auseinanderzusetzen. Ich kann unmöglich von Yvorne aus mich in all diesen Dingen einmischen. Sie müssen Ihre oft zu intensiven Sympathie- 
u. besonders Antipathiegefühle überwinden, und ruhig und objektiv auch mit solchen Menschen unterhandeln, die Ihnen zuwider sind.

An Ihrer Stelle, statt zu schmollen, würde ich gelegentlich mit den Burghölzlianern sprechen und discutieren und ihnen ev. ihre Fehler nachweisen.

Unter allen Umständen ist es aber ein Jammer, wenn Sie und Frank die ihr beide die psychanalytische Methode so ausgebildet habt hintereinander kommt, resp. daß Sie dem Frank ohne Grund so mißtrauen. Das ist für die objective Sache schlecht, für Schloß Hard schlecht, für die Kranken nicht gut.

Ich bitte Sie daher inständig, überwinden Sie Ihre Gefühle und setzen Sie sich einmal gründlich und freundschaftlich mit Frank auseinander über die ganze Sache u. auch über die Candidaturen für Schloß Hard. Es ist natürlich schon etwas mißlich einen in der thurgauischen Anstalt angestellten Arzt so direct wegzurufen, wenn er sich nicht meldet, umsomehr als Schl. H. im Thurgau ist. Übrigens kenne ich Hans Mayer gut u. ist er ein sehr netter Kerl, den Sie sicher sehr gut abrichten können. Somit braucht man sich da nicht so zu echauffieren. Eine ruhige Aussprache wäre viel besser.

Also bitte um baldige Antwort bezüglich Herrn $\mathrm{H}$.

\section{Herzlichen Gruß von Haus zu Haus Ihr Forel}

Es wird nicht klar, ob es sich bei dem genannten Hans Mayer um den späteren Nachfolger E. Bleulers im Burghölzli handelt. H. = Patient von Forel.

\section{Maschinengeschriebener Brief}

Yvorne, d. 22.XI.07

Mein lieber Herr Kollege,

Besten Dank für Ihren Brief. Wir warten also auf das Telegramm um Herrn Hervé zu Ihnen zu schicken.

Ich bin mir aber sicher bewußt, Ihnen kein Unrecht getan zu haben. Ich habe Ihnen bloß die Bemerkungen Frank's und seinen Wunsch um eine Auseinandersetzung mitgeteilt. Wenn das unrecht heißt, so weiß ich nicht mehr was recht ist. Es fällt mir gar nicht ein, gegen irgend jemanden parteiisch zu sein oder Ihre Objektivität zu bezweifeln. Ich bin auch romanisch und kann daher recht gut mit Ihnen fühlen. 
Ich werde sehr gerne Ihre Kontroverse lesen, wenn Sie sie mir schicken. Ich bin ja von vorne herein auf Ihrer Seite in der ganzen Sache. Frank ist sogar mit mir nicht besonders zufrieden. Das einzige worin ich Ihnen nicht ganz beistimme, ist die Taktik des Sichzurückziehens. Ich glaube daher heute noch, als richtiger Freund gehandelt zu haben, indem ich Ihnen von dieser Taktik abrate. Sie brauchen deshalb bei Leibe keinem Freudklub beizutreten. Mir selbst ist der Freud ja selbst höchst unsympatisch, aber ich glaube Sie werden in Ihrer Stellung mehr erreichen, wenn Sie sich mit Frank in aller Ruhe, frank und frei auseinandersetzen und wenn Sie gelegentlich mit den Freudnarren einen Strauß ausfechten, als wenn Sie ihnen aus dem Weg gehen.

Ich selbst bin leider infolge meiner vielseitigen Hetze nicht im Stande die Jung'schen Arbeiten zu verfolgen. Ich traue dem Jung nur halb und kann nicht à distance beurteilen, was an seinen eigenen Leistungen objektive Wahrheit und subjektive Täuschung ist. Um so wichtiger wäre es, wenn eine Person wie Sie, der Sie so stark in diesem Fach eingearbeitet sind, mit möglichster Eindämmung des romanischen Blutes (ich versuche es auch immerwährend an mir selbst!) sich daran macht mit Jung zu diskutieren und seine Ergebnisse nachzuprüfen. Sie werden sicher nur Gewinn ziehen, sei es positiv, sei es negativ, Gewinn bleibt es stets für das objektive Wissen. Wäre ich in Zürich und wie gesagt nicht so furchtbar abgehetzt, so würde ich es tun.

Ich empfehle Ihnen dringend nochmals den Herrn H. Er ist ein ganz vorzüglicher Mensch, von scharfem Verstand und hohem ethischen Wert. Er ist ein durch und durch logischer und klarer philosophischer Kopf. Sie werden an ihm Ihre Freude erleben. Es ist ein Jammer, daß ein solcher Mensch durch Zwangsvorstellungen lahm gelegt wird. Wenn Sie ihn heilen, haben sie eine große Tat vollbracht.

Ich ärgere mich furchtbar über mich selbst, daß meine Ungeschicklichkeit und mein Mangel an Übung mir keine Komplexe zu entdecken gestatten. Bei mir wollen die Leute keine verraten, trotz allen Lehren die ich von Ihnen und Frank empfangen habe.

Selbstverständlich werde ich mit großer Freude mit Ihnen sprechen. Aber wie, wo und wann? Daß ich in nächster Zeit in Ihre Gegend komme, davon ist keine Rede; ich bin Zeit und Kraft bankrott auf der ganzen Linie und Sie wohl auch? Jedenfalls würde es uns freuen, Sie hier zu empfangen. Wir würden Sie schon logieren können, und dann würde ich Ihnen meine Fourmilière zeigen. 
Mein lieber Freund und Kollege,

Ich habe eine geniale «Idee» gehabt, nämlich an die Quelle, direkt an Breuer zu gehen mit dem ich gut stehe und habe in frank und frei gefragt, welchen Anteil er an der Psychanalyse habe; die bezügliche Methode werde jetzt von Bezzola, Frank und andern mit großem Erfolg angwendet; ich interessiere mich dafür und es sei mir eine Gewissenssache genau zu wissen was ihm und was Freud gehöre um niemandem Unrecht zu tun.

Ich kenne Breuer als einen außerordentlich vorsichtigen und gewissenhaften Menschen, der sich selbst nicht im mindesten überschätzt, außerordentlich kritisch ist und gerade deshalb weil er mit Freud entzweit ist, sich peinlich davor hüten wird irgend etwas für sich in Anspruch zu nehmen was nicht ihm gehört. Beiliegend seine köstliche Antwort. Darin erkennen Sie den Mann, aber auch den Vorsichtspeter der nicht ein Wort gegen Freud sagt. Man muß zwischen den Zeilen lesen. Der Brief ist natürlich konfidentiell, aber immerhin so gehalten, daß ihn Freud direkt lesen könnte ohne ein Wort dagegen sagen zu können. Ich ersuche Sie mir ihn wieder zu senden. Ich gestatte Ihnen jedoch eine Abschrift davon zu nehmen. Sie dürfen sich aber darauf beziehen, wenn man Sie in der Sache schikanieren sollte. Jedenfalls gibt es Ihnen eine vortreffliche Handhabe und ich freue mich Ihnen dieses Dokument zeigen zu können. Die Adresse Breuer's ist I., Brandstätte, 4, Wien, für den Fall wo Sie wegen Ihren Studien mit ihm korrespondieren wollen.

Ich warte also auf Ihr Telegramm bezüglich Herrn Hervé.

Mit herzlichstem Gruß, Ihr Forel

Der erwähnte Brief Breuers findet sich abgedruckt bei Walser ${ }^{8}$ (S. 395).

23. Aus einem Brief Dr Bezzolas an den Direktor Dr. Frank:

Ermatingen, 26. Nov. 1907

Ich bin anstaltsmüde und satt und habe außerdem das dringende Bedürfnis das fast zum Gewissenszwang wird meine hiesigen Erfahrungen zum Kampfe gegen die Freud'schen 
Verirrungen auszurüsten. Zu diesem Zweck brauche ich 6 Monate Ferien oder völlige Loslösung vom Schloß Hard. Ersteres wird dem Verwaltungsrat nicht passen wie Sie sagen. Es bleibt mir nur noch der zweite Ausgang.

Daher habe ich mich entschlossen auf den 1. Juni 1908 zu kündigen. Ich kann nicht anders wenn ich nicht neue Konflikte heraufbeschwören und dadurch unser jetzt frisch geordnetes persönliches Verhältnis wieder stören will.

Ihr ergebener D. Bezzola

\section{Maschinengeschriebener Auszug aus Brief Bezzolas vom 9.IV.08 an A. Forel}

Eine ältere (1860) unverheiratete Hysterica wurde mir Mitte November aus Holland zur analytischen Behandlung zugewiesen. Hauptbeschwerden: Schmerzen \& Paraesthesien, die sich speziell an Gemütsbewegungen anknüpfen \& formell zum Teil von einer Nephrographie herrühren. Der betr. Operateur hat längere Zeit mehr weniger platonische Beziehungen mit der Pat. unterhalten \& sie auch längere Zeit hypnotisiert. Einmal wurde sie mittelst Kuß aus der Hypnose geweckt. Vermutlich handelt es sich um sexuellen Mißbrauch der suggestiven Gewalt oder dann um einen hysterischen Traum mit Übertragung auf den betr. Arzt. Ersteres ist nach dem Inhalt der Korrespondenz zwischen den beiden wahrscheinlicher. Die Analyse hat bis jetzt keinen rechten Erfolg gehabt, vermutlich weil ich nicht recht wage, den hypnotischen Zustand herbeizuführen \& ohne diesen, das eigentliche Trauma nicht ins Bewußtsein tritt. Der betr. Arzt soll ihr auch die Suggestion gegeben haben, daß andere Leute sie nicht hypnotisieren können.

Nun trete ich nach Ostern meine langersehnten Ferien an \& mein neuer Assistenzarzt Dr de Montet ist noch zu wenig eingelebt, um so schwierige Fälle übernehmen zu können. Daher möchte ich Sie, lieber Herr Professor, fragen, ob die betr. Dame während meiner Abwesenheit von Schloß Hard (Ostern-Pfingsten) von Ihnen hypnotisch behandelt werden könnte. Wenn ich mich nicht irre, ist in der Nähe Ihrer Fourmilière eine geeignete Pension, wo Kranke Verpflegung finden. Die Hauptindikation zur hypnot. Behandlung wäre eine Gewöhnung an den betr. Zustand d.h. die Aufhebung der hypnosenfeindlichen Suggestion des betr. Arztes. Daneben wäre es wünschenswert, ihre enorme Hyperalgesie zu heben \& ihr einen Weg für die Zukunft anzugeben. Ihr Leben ist leider vollständig ziellos \& ich glaube, daß eine Ihrer großen sozialen Zielsuggestionen der Hauptfaktor einer Heilung sein könnte, wenn die Begeisterungsfähigkeit nicht lediglich Strohfeuer ist.

\section{Postkarte}

10.04 .1908

Forel berichtet über die vorgenannte Patientin. 


\section{Eigenhändiger Brief}

Forel berichtet über eine Patientin.

\section{Postkarte}

Forel berichtet nochmals über die Suggestionsbehandlung dieser Patientin. Ferner: «Besten Dank für die Bierzeitung. Soll ich sie zurückschicken? Wenn sie nichts schreiben, behalte ich sie. Prof. Monitor ist doch Dubois und Lustmexer Freud, oder? Wer hat die trefflichen Witze gemacht?»

\section{Eigenhändiger Brief}

Yvorne, 21.09.1908

Forel überweist eine Patientin.

Postscriptum: Ich habe jetzt einen Fall (durch Hypnose) in Behandlung der durch Psychoanalyse v. Freud u. Schule total kaputt gemacht worden ist. Aus lauter «sexuellen» Deutungen aller harmlosesten Dinge ist die betreffende halb toll geworden. Ich glaube es gibt eine Art Psychoanalyse die mehr Komplexe erzeugt als sie solche beseitigt! [...]

\section{Eigenhändiger Brief}

Yvorne, 3.10.1908

Forel bittet B., seine Arbeit über die Idiotenkurve an eine Ärztin zu schicken.

\section{Eigenhändiger Brief}

«Ich bin an der Grenze menschlicher Leistungsfähigkeit und in einer Weise überarbeitet die jeder Beschreibung spottet» [...]

\section{Eigenhändiger Brief}

Forel schreibt über eine Patientin, die er bei sich nicht aufnehmen könne und die Bezzola übernehmen könnte. «Es ist nur eine eventuelle Anfrage für den Fall, daß ich mit Hypnose nicht auskomme und eine Psychanalyse mir indiziert erschiene. [...] 
Neulich habe ich mit Hypnose in 2-3 Wochen einen Fall geheilt, der monatelang von Freud und Jung psychanalytisch verschlimmert (sexuelle Deutungen und Complexfabrik) worden war.»

\section{Vervielfältigtes Zirkularschreiben}

Yvorne, Schweiz, Datum des Poststempels

Sehr geehrter Herr Kollege,

Einerseits wird die Psychotherapie in den medizinischen Fakultäten der Hochschulen völlig vernachlässigt; andererseits besteht sie in ganz zerstreuten individuellen Bestrebungen, die ohne Zusammenhang untereinander bleiben. Nur selten findet man in Kongressen Gelegenheit über die bezüglichen großen Fragen: Hypnotismus, Suggestion, Psychanalyse, etc. zu diskutieren, da jene Versammlungen sich mit anderen Gegenständen beschäftigen. Wenn man davon spricht, wird man mit einem ironischen oder ungläubigen Lächeln empfangen, weil die bezüglichen Kollegen von der Sache nichts verstehen.

Ich habe mich neulich in unserem Journal für Psychologie und Neurologie über die Sache beklagt und gesagt, es wäre nötig die Bestrebungen derjenigen Personen zu vereinigen, die sich mit medizinischer Psychologie und mit den verschiedenen Arten der Psychotherapie beschäftigen. Die Frage ist wichtig genug. Herr Kollege Dr Oscar Vogt in Berlin und ich sind der Überzeugung, es wäre nützlich in Form eines Vereins die Ärzte zu gruppieren, die den ungeheuern Einfluß und die Heilwirkung begriffen haben, welche die Gehirndynamik auf die ganze Ökonomie unseres Körpers übt. Auf diese Weise könnten die Mitglieder in wissenschaftlichen und medizinischen Kongressen eine Gruppe, eventuell eigene Sektionen bilden und ihre verschiedenen Methoden und Kenntnisse austauschen.

Wir haben uns daher entschlossen, einige Personen, unter welchen Sie sich befinden, zu einer Sitzung einzuberufen, die in Salzburg bei Anlaß der Jahresversammlung deutscher Naturforscher und Ärzte von 19. bis 25. September 1909 stattfinden wird, um die eben erwähnte Frage zu besprechen und nötigen Falls besagten Verein zu gründen. Wenn Sie selbst nicht anwesend sein können, werden wir Ihnen sehr verbunden sein, uns wenigstens Ihre schriftliche Zustimmung zu geben und uns zugleich zu sagen, ob Sie den beiliegenden Statutenentwurf gutheißen oder umgekehrt Änderungen daran wünschen. 
Selbstverständlich müssen wir uns sowohl gegen die Charlatanerie als gegen die Verwirrungen schützen, die von occultistischer Seite oder durch metaphysische Theorien gestiftet werden. Derartige Bestrebungen sind bekanntlich stets bereit, unsere wissenschaftlichen Bestrebungen zu überwuchern und sie in Mißkredit zu bringen.

Genehmigen Sie, verehrtester Herr Kollege, den Ausdruck unserer vorzüglichsten Hochachtung und Ergebenheit.

\section{Stempel: Dr August Forel Professor}

Forel war ein begeisterter Vereinsgründer, und auch hier zeigt sich die Tendenz, Gleichgesinnte zu vereinigen. Trotzdem hatte diese Initiative Pioniercharakter.

33. Nachgesandtes Zirkular zum Schreiben vom 19.08.1909

Die Vorbesprechung zur Gründung eines Internationalen Vereins für medizinische Psychologie und Psychotherapie findet in Salzburg am 22. September 1909 abends 8 Uhr statt. Das Lokal wird dort bekannt gegeben.

\section{Handschriftlicher Brief Bezzolas}

Lieber Herr Professor,

Nach dem stillen Begräbnis meiner Tätigkeit in Schloß Hard kommt mir als erstes Lebenszeichen Ihrerseits das Circular betr. Gründung eines internationalen Vereins für medizinische Psychologie und Psychotherapie zu. Diese Anregung zu einer wirklich notwendigen Veranstaltung freut mich umsomehr als ich sehe, daß Sie meinen rein praktischen Erfahrungen, die ich eben zur Bekämpfung der Freudschen Dogmen zusammenstellte, einige Wichtigkeit beimessen. Ich hoffe im Laufe dieses Winters mit einer größeren Publikation fertig zu werden, die vor allem den Zweck hat, die Suveränität der Seele gegenüber fremden Deutungen ihrer Ausdrucksmittel zu verteidigen und den Einwendungen der Freud'schen Schule zu begegnen, daß ich den «Complexen» nicht auf den Grund gehe. Insbesondere muß ich die immer wiederkehrende, auch von anderer Seite (Frank in Amsterdam) aufgestellte Behauptung ad absurdum führen, als ob wir Freud seine spezielle Betonung des Sexualtriebs übel nähmen. Wir bekämpfen nur seine dogmatische Ausschließlichkeit und die Aufoktroyierung seines eigenen Hirnmechanismus. Die Traumdeutung von Freud ist subjektive Wahrheit, darf aber 
nicht zum Katechismus der Psychologie werden und die Gefahr liegt unbedingt vor wie die Zürcher Schule beweist. Ich sträube mich auch dagegen, wenn jemand behauptet, ich sehe etwas als Esel an was ich unterbewußt für einen Ochsen halten sollte, weil sein eigenes Unterbewußtsein alle Esel für Ochsen hält. Warum sollte ich mich nicht dagegen wehren, Geldbörsen als Vaginae und Türklinken als Penis anschauen zu müssen? Das Unanständige liegt in der Insinuation und nicht in der Sexualität. Das wird meiner Ansicht nach zu wenig betont und es handelt sich doch um die Suveränität der Seele.

Es tut mir leid am 22. September nicht nach Salzburg kommen zu können, weil ich hier an die Saison gebunden bin, die möglicherweise noch nicht fertig ist. Mit Ihren Statuten und der Gründung bin ich vollständig einverstanden und erkläre mich bereit, als Mitglied des neuen Vereins im Sinne seiner Bestimmungen zu leben.

Ich hätte speziell Sie, lieber Herr Professor, gerne einmal wieder gesehen und mich überzeugt, daß Sie meine Flucht aus Schloß Hard nicht übel genommen haben. Nicht daß ich sie bereue; denn die dortigen Arbeitsbedingungen waren mir schon längst unerträglich geworden und meine Reizbarkeit fing massive an dabei mich in Konflikte zu verwickeln. Ich bereue im Gegenteil, daß ich zu lange geblieben bin, als ich nach meinem Weggang bemerkte was ich alles in dieser Arbeits... (unleserlich) eingebüßt habe. Fürchten Sie nur nicht, daß ich zu Ihnen komme um mich zu beklagen! Ich möchte Sie bloß um die Erlaubnis bitten, Sie diesen Herbst z. B. im Oktober einmal besuchen zu dürfen, um Ihnen Red und Antwort zu stehen, falls Sie sich über mich zu beklagen haben. Es wäre mir peinlich Ihnen, dessen Vertrauen ich für das Beste halte was ich besitze, meine psychotherapeutische Erfahrung verdanke, als undankbar zu erscheinen. Bei dieser Gelegenheit kann ich Ihnen auch meine Zukunftspläne vorlegen, falls Sie sich dafür interessieren. Sind Sie im Oktober daheim?

Empfangen Sie und Ihre liebe Familie herzlich hochachtungsvolle Grüße von meiner Frau und Ihrem stets ganz ergebenen

Bezzola

Die «größere Publikation», von der B. in diesem Brief schreibt, scheint nicht zustandegekommen sein.

\section{Maschinengeschriebene Karte}

Yvorne, d. 25.08.09

Mein lieber Kollege,

Zunächst bin ich froh, Ihre Adresse zu haben. Ich wußte nicht in welchem Weltteil Sie waren. Zweitens haben Sie sich gar nicht zu rechtfertigen und habe ich mich gar nicht über Sie zu beklagen; ich war nicht dort und kann die Sache nicht beurteilen. Ich freue mich sehr, daß Sie an die Publikation Ihrer Ergebnisse gehen. Hoffentlich sprechen Sie sich auch deutlich über die Methode aus. Bleiben Sie in Zuoz? denn ich möchte wissen, ob und wo ich 
Ihnen eventuell Patienten zuweisen kann. - Es freut mich, daß Sie mitmachen wollen. Ich wirke nur als Schnürlimann, weil ich die Sache wirklich für dringend nötig halte. Selbstverständlich werde ich Sie sehr gerne im Oktober hier empfangen. Im September bin ich eben abwesend. Freilich müssen Sie vorher das Datum anmelden, da ich im Oktober verschiedene Male Vorträge im Kanton Waadt zu halten habe.

Mit herl. Gruß, Ihr ergebenster Forel

36. Schreibmaschinenbrief

Yvorne, 17.05.1910

Lieber Herr Kollege,

Herr H. hat mich soeben besucht und ich habe mich gefreut, über die riesigen Fortschritte, die er gemacht hat. Sehr betrübt hat es mich dagegen zu hören, daß es Ihnen gar nicht gut geht, daß Sie sich überarbeitet haben und daß Sie auch infolge einer großen Erkältung mit den Lungen zu tun haben. Ebenso leid tut es mir, daß Sie noch immer nicht dazu kommen, Ihr Buch über Ihre Erfahrungen zu schreiben. Letzteres ist aber ein dringendes Bedürfnis. Die ganze Frage wird durch Freud und seinen Anhang total verfuhrwerkt und diskreditiert. Es ist höchste Zeit, daß die besonnenen und wissenschaftlichen Psychanalytiker durch ein größeres und ernstes Werk eingreifen.

Ich bitte Sie dringend, im Namen unserer Wissenschaft und der Menschheit nicht länger zu zögern. Wie ich durch H. höre, haben Sie in St. Moritz längere Ferien, und ich bin überzeugt, daß Ihnen eine solche Arbeit noch Freude machen würde. Bitte gehen Sie ans Werk. In der Person H. werden Sie einen Freund haben, der Ihnen eine französische Ausgabe nach feinstem und bestem französischen Styl ausfeilen wird. So könnten Sie in beiden Gebieten bahnbrechend wirken. Es ist ein wahrer Jammer, daß Sie damit schon so lange gezögert haben. Ich bitte nochmals flehentlich, tun Sie es nicht länger.

Was nun Ihre Gesundheit anbetrifft, so möchte ich Sie ebenso dringend bitten, sich gründlich untersuchen zu lassen, und sich eventuell, wenn etwas Verdächtiges da wäre, durch die Immunkörpertherapie Karl Spenglers behandeln zu lassen. Unsere Freundin, Frau Professor Fuchs, Villa Kaiser, 
Davos-Platz, würde Ihnen sicher darin in allen Beziehungen entgegen kommen.

Mit herzlichstem Gruß von uns allen an Sie und Ihre Frau Gemahlin.

A. Forel

\section{Handschriftlicher Brief Bezzolas}

Bezzola dankt für den erhaltenen Brief, beruhigt Forel hinsichtlich seines Gesundheitszustandes, teilt mit, daß seine Frau Freude habe, die neue Wohnung einzurichten. Kündigt an, daß er nun Zeit haben werde, sein Material zu sichten. [...] « die Notwendigkeit habe ich wieder einmal in Herisau deutlich fühlen können, wo Dr Jung in seinem Vortrag über Symbolik eine Menge (psychologischer Wahrheiten) verzapfte, ohne auch nur einen inductiven Beweis zu führen. Auf eine Diskussion verzichtete ich, weil Glaubenssätze sich nicht diskutieren lassen. [...]

Seit Anfang April analysiere ich täglich 3-4 Stunden und ich muß um das liebe tägliche Brot auf diesem Pflaster arbeiten. [...]»

\section{Maschinengeschriebener Brief}

Lieber Freund und Kollege,

Ihr Brief erfüllt mich mit großer Freude. Gerade heute schicke ich Ihnen einen Patienten, der schon mit Ihnen correspondiert hat (Dr H.).

Ihr Brief gibt mir nun zu folgender Frage Mut: Würden Sie uns nicht im Verein für medizinische Psychologie und Psychotherapie in der Sitzung in Brüssel am 7. u. 8. August einen Vortrag über Psychanalyse halten? Die Freudianer versagen, ebenso Frank und Bleuler. Frank ist offenbar durch die Schloß-Hardgeschichte stark angegriffen. Könnten Sie sich nicht entschließen, für zwei Tage abzukommen? Ich werde dort sein, ebenso Vogt, Janet, und andere Größen. Die Gelegenheit wäre einzig einmal wissenschaftlich in der ganzen Sache das wahre Korn vom Mist zu scheiden. Ich würde mich furchtbar freuen, wenn Sie zusagen würden und wir haben so etwas dringend nötig. Ihre Patienten können ja während Ihrer Abwesenheit ein paar Bergtouren machen und der guten wissenschaftlichen Sache wäre ein großer Dienst erwiesen.

Mit herzlichstem Gruß,

Ihr getreuer

A. Forel 
Lehnt die Einladung Forels, in Brüssel zu sprechen mit dem Hinweis ab, daß er zuerst sein Material sichten möchte. Er will auch die Semonschen Forschungsresultate, «speziell die in den mnemischen Empfindungen niedergelegten mit den meinigen vergleichen».

\section{Postkarte}

Yvorne, 6.06.1910

Lieber Freund und Kollege,

Es tut mir leid, daß Sie nicht nach Brüssel können, wir werden Sie schwer vermissen. Aber ich begreife Sie und dränge nicht weiter, umsoweniger als de Montet uns in der Tat das Leben rettet, nachdem uns alle andern Psychanalytiker im Stiche ließen. Was Sie mir über Semon's «Mnemische Empfindungen» schreiben, interessiert mich im höchsten Grade und ich gestatte mir, Ihnen mein bezügliches Referat zu senden. Es wird Semon furchtbar freuen, wenn Sie ihm schreiben. Seine Adresse ist: Martiusstraße 7, München 23.

Herzlichsten Dank für Ihr sehr interessantes Separatum.

Mit herzlichsten Grüßen an Sie und die Ihrigen.

Ihr ganz ergebener Forel

41. In einem vervielfältigten Zirkular lädt Forel abstinente Schweizer Ärzte, darunter auch Bezzola ein, eine eigene Schweizer Gruppe zu gründen.

Auch hier bemerkt man wiederum den missionarischen Eifer Forels für «Gründungen». Bezzola teilt kurz sein Einverständnis mit.

42.

Yvorne, 30.01.1911

Lieber Herr Kollege,

Für die jetzt von mir umgearbeitete VI.Auflage meines Hypnotismus schreibe ich ein Kapitel über Psychanalyse. Ich möchte recht gerne Ihre 
demnächst erscheinende Arbeit wenigstens dem Titel und dem Verlag nach erwähnen, resp. darauf hinweisen, um meine Leser auf die bezüglichen objektiven Tatsachen aufmerksam zu machen. Hätten Sie deshalb wohl die Güte, mir den genauen Titel dieser Arbeit und wenn möglich den Verlag anzugeben, sowie die Zeit wann sie ungefähr erscheinen wird?

Im Voraus bestens dankend u. besten Gruß von Haus zu Haus.

Ihr ergebenster Forel

In seinem Buch über Hypnose (6. Auflage 1918) erwähnt Forel Bezzola nicht.

\section{Postkarte}

Yvorne, 6.1.1911

\section{Lieber Herr Kollege,}

Ich danke Ihnen herzlichst für Ihren interessanten Brief. Es handelt sich bei mir nur darum, ob Sie einverstanden sind, daß ich eine Andeutung über Ihr in Aussicht stehendes Buch mache. Ob es etwas früher oder später erscheint, tut schließlich nicht viel zur Sache, nur muß ich einen ungefähren Titel angeben können. Wenn Sie somit nichts Weiteres schreiben, nehme ich an, Sie seien einverstanden, und schreibe als Titel: «Das Psychotrauma, analytisch psychopathologische Studien». Was den Verlag anbetrifft, so ist mir heute, offen gestanden, Reinhardt in München lieber als Enke, der ein alter, vornehmer, bequemer Herr ist. Sie können übrigens bei beiden und noch Andern um ihre Bedingungen anfragen und die günstigsten nehmen.

\section{Postkarte}

Yvorne, 4.Sept. 1916

Lieber Freund und Kollege,

Vor lauter Huldigungen an meinem Geburtstage sinke ich fast um. Ich kann Ihnen nur kurz für Ihren schönen Aufsatz im Band 43 der Zeitschrift für die 
gesamte Neurologie \& Psychiatrie von ganzem Herzen danken, den Sie mir gewidmet haben.

\author{
Mit herzlichsten Grüßen \\ von Ihrem alten Freund und Kollegen \\ Dr A. Forel
}

Mit diesem Dankesbrief hört die Korrespondenz zwischen August Forel und Dumeng Bezzola auf.

Die hier veröffentlichete Korrespondenz beleuchtet in eindrücklicher Weise die Lage, in der sich August Forel nach seinem Rücktritt vom Lehrstuhl für Psychiatrie in Zürich befand. Sein vorzeitiges Ausscheiden aus der offiziellen Psychiatrie hat zu mancherlei Spekulationen Anlaß gegeben. Handelte es sich um einen reiflich überlegten Entschluß oder um eine übereilte Entscheidung als Resultat eines persönlichen Unmutes über die Situation in Zürich? Die Korrespondenz mit Bezzola gibt uns darüber keine Auskunft. Deutlich wird indessen, wie sehr August Forel seinem Nachfolger Eugen Bleuler grollte, der sich für Freud einsetzte und außerdem die klare Trennung von Neurologie und Psychiatrie forderte (siehe dazu Briefband Walser).

Ein wichtiges Element für das Verständnis der Lage der Psychiatrie jener Jahre tritt deutlich hervor: das Aufbrechen des Interesses an der Psychotherapie ganz allgemein. Forel empfand sich offensichtlich als Vorkämpfer der Psychotherapie, interessierte sich brennend für deren Fortschritte und fand in Bezzola einen 20 Jahre jüngeren aktiven Mitstreiter. So wundert es nicht, daß er ihm mit Vorliebe Patienten schickte.

Ferner taucht in dieser Korrespondenz ganz besonders eindrücklich die negative Einstellung Forels gegenüber der Freud'schen Psychoanalyse auf. Wie Eugen Bleuler auch, ist Forel zwar bereit, sie «objektiv» zu prüfen, lehnt sie aber dann doch in einer wunderlichen Ambivalenz ab. Einerseits versucht er die psychoanalytische «Technik» in seiner Praxis anzuwenden, wobei er sich etwas naiv auf das «Herausholen» von Reminiszenzen konzentriert, andererseits lehnt er Freuds theoretischen Ansatz ab. Er wettert in derben Worten gegen die angeblich Freud verfallene Zürcher Clique, und weder Bleuler noch C.G.Jung werden geschont. Es ist nicht von der Hand zu weisen, daß dabei neben grundsätzlichen theoretischen Überlegungen auch ein Generationenkonflikt eine maßgebende Rolle gespielt haben mag. Es handelt sich gewissermaßen um das Drama des älteren, berühmten Arztes, 
der auf der Höhe bleiben möchte und doch die neuen Tendenzen nicht bejahen kann. Forel kann es seinem jüngeren Nachfolger nicht verzeihen, daß er andere Wege einschlägt, als er selbst vorgezeichnet hatte. Da bis heute noch keine umfassende Biographie Eugen Bleulers vorliegt, wissen wir wenig darüber, wie Bleuler diesen Konflikt erlebt hat.

Im übrigen erscheint Forel in dieser Korrespondenz als ein hyperaktiver, ja gehetzter Schwerarbeiter. Seine Organisierlust und sein missionarischer Kampf gegen den Alkoholismus werden durch den Briefwechsel verdeutlicht. Bezzola hat sich ganz auf Forels Seite gestellt und ihm Schützenhilfe geboten. Wie sehr der Konflikt zwischen Frank und Bezzola, die Forel wohl als seine «Schüler» betrachtete, ihn belastete, geht ebenfalls aus der Korrespondenz hervor.

Der temperamentvolle, kämpferische Einsatz Forels für eine Idee, wie er in diesen Briefen zum Ausdruck kommt, beleuchtet und ergänzt unser Wissen um die Persönlichkeit dieses großen Schweizer Psychiaters. Wir können uns W. Leibbrand anschließen, der im Klappentext des Buches von A. Wettley schrieb: «Nicht Sexus, nicht Hypnose, nicht Abstinenz ist hier die Frage. Sie alle dienen nur der Verwirklichung der monistischen Idee. Die Analyse dieser Entwicklung ist fesselnd und atemberaubend. Wie die Peripetie eines Dramas wirkt schließlich der irrationale Abgang des Weltapostels und vermeintlichen Gegners des Christentums».

\section{Literaturverzeichnis}

1 D. Bezzola: Statistische Untersuchungen über die Rolle des Alkohols bei der Entstehung des originären Schwachsinns. Verhandlungen des VIII. Internationalen Kongresses gegen den Alkoholismus, Wien, 1901.

2 D. Bezzola: Zur Analyse psychotraumatischer Symptome. Journal f. Psychol. u. Neurol. $8,1907$.

3 D. Bezzola: Elementar-Autanalyse (eine kritisch-methodologische Studie). Z. f. d. ges. Neurol. u. Psych. 43, Heft 1/2, 1918, S.27-33.

4 F. Braun: Dr. med. Dumeng Bezzola (1868-1936) Nekrolog. Schw. Arch. f. Neurol. u. Psych., Bd. 38, Heft 2, 1936, S. 360-364.

5 A.Forel: Der Hypnotismus oder die Suggestion und die Psychotherapie. 7. Aufl. Stuttgart, F. Enke, 1918.

6 A. Forel: Rückblick auf mein Leben. Zürich, Europa Verlag, 1935.

7 W. McGuire und W.Sauerländer (Hrsg.): Sigmund Freud-C.G. Jung Briefwechsel. Frankfurt, S. Fischer, 1974. 
8 H. Walser (Hrsg.): August Forel. Briefe. Correspondance 1864-1927. Bern, H. Huber, 1968.

9 A. Wettley: August Forel. Salzburg, O. Müller, 1953.

\section{Summary}

\section{August Forel and Dumeng Bezzola - the correspondence}

The correspondence between August Forel (1848-1931) and Dumeng Bezzola (1868-1936), a forgotten Swiss psychiatrist, shows the deep ambivalence of Forel in regard of Freudian theories. Forel expresses clearly his scepticism not only concerning psychoanalysis in itself but also in regard of the Burghölzli School, i. e. Eugen Bleuler and Carl Gustav Jung. Forel and Bezzola were both involved in the vigorous fight against alcoolism.

\section{Résumé}

Auguste Forel et Dumeng Bezzola. Correspondance éditée et commentée par Christian Müller.

Cette correspondance (1901-1916) entre Auguste Forel et Dumeng Bezzola (1868-1936), un psychiatre aujourd'hui quelque peu oublié, révèle la position ambiguë de Forel à l'égard de Freud. Elle touche également au successeur de Forel au Burghölzli de Zurich, Eugen Bleuler, et à son collaborateur Carl Gustav Jung, qui tous deux se sont efforcés d'intégrer la psychanalyse au traitement des maladies mentales.

Prof. Dr. med. Christian Müller

Herrengasse 23

CH-3011 Bern 